\title{
On Multiple Zeta Function and Associated Properties
}

\author{
Maged G. Bin-Saad ${ }^{1, *}$, M. A. Pathan ${ }^{2}$, Ali Z. Bin-Alhag ${ }^{1}$ \\ ${ }^{1}$ Department of Mathematics, Aden University, Aden, Kohrmaksar, P. O. Box 6014, Yemen \\ ${ }^{2}$ Centre for Mathematical and statistical Sciences (CMSS), KFRI, Peechi P.O., Thrissur, Kerala-680653, India \\ *Corresponding author: mgbinsaad@yahoo.com
}

Received January 01, 2018; Revised April 06, 2018; Accepted May 28, 2018

\begin{abstract}
The purpose of this paper is to introduce and investigate a new class of multiple zeta functions of $n$ variables. We study its properties, integral representations, differential relation, series expansion and discuss the link with known results.
\end{abstract}

Keywords: multi-variable zeta functions, Hurwitz-Lerch Zeta function, Hyper-geometric function, Lauricella functions, Summation formula, expansion formulas

Cite This Article: Maged G. Bin-Saad, M. A. Pathan, and Ali Z. Bin-Alhag, "On Multiple Zeta Function and Associated Properties." Turkish Journal of Analysis and Number Theory, vol. 6, no. 3 (2018): 84-89. doi: 10.12691/tjant-6-3-3.

\section{Introduction} $[1,2]$

The generalized (Hurwitz's) zeta function is defined by

$$
\zeta(s, a)=\sum_{n=0}^{\infty}(a+n)^{-s}(a \neq 0,-1,-2, \ldots ; \Re(s)>1),(1.1)
$$

so that when $\boldsymbol{a}=1$, we have

$$
\zeta(s, 1)=\sum_{n=0}^{\infty} n^{-s}=\zeta(s)
$$

where $\zeta(s)$ is the Riemann zeta function. The function $\Phi(x, s, a)$ extends (1.1) further, and this generalized Hurwitz-Lerch zeta function [[1], p. 316], is defined by

$$
\Phi(x, s, a)=\sum_{n=0}^{\infty} \frac{x^{n}}{(a+n)^{s}},
$$

$(a \neq 0,-1,-2, \ldots,|x|<1 ; \Re(s)>1)$, when $|x|=1) ?$

A generalization of (1.3) is the Zeta function $\Phi_{\mu}^{*}$ which is defined by [[3], p.100, (1.5)]:

$$
\Phi_{\mu}^{*}(x, s, a)=\sum_{n=0}^{\infty} \frac{(\mu)_{n ?} x^{n}}{(a+n)^{s} n !}
$$

$\mu \in \mathbb{C} ; a \in \mathbb{C} \backslash \mathbb{Z}_{0}^{-} ;|x|<1$, where $(\mu)_{n}=\frac{\Gamma(\mu+n)}{\Gamma(\mu)}$, for $n=0,1,2, \ldots$, denotes the Pochhammer's symbol and $\Gamma$ denotes the Gamma function. Evidently, we have

$$
\Phi_{l}^{*}(x, s, a)=\Phi(x, s, a)
$$

$$
\begin{gathered}
\Phi(1, s, 1)=\zeta(s), \\
\Phi(1, s, \boldsymbol{a})=\zeta(\boldsymbol{s}, \boldsymbol{a}) .
\end{gathered}
$$

The zeta functions in (1.3) and (1.4) have since been extended and generalized by a number of workers (see e.g. [3-14]. The present sequel to these earlier papers is motivated largely by the aforementioned works of Matsumoto and Kamano $[15,16]$ in which the zeta function $\zeta(s, a)$ in (1.1) was generalized to the following multiple Hurwitz zeta function

$$
\zeta_{n}\left(s_{1}, \ldots, s_{n} ; a\right)=\sum_{m_{1}, \ldots, m_{n}=0}^{\infty} \frac{1}{\left(a+m_{1}\right)^{s_{1}} \ldots\left(a+m_{n}\right)^{s_{n}}},
$$

$\left(a>0 ;\left(m_{1}, \ldots, m_{n}\right) \in \mathbb{Z}^{n}, 0 \leq m_{1}<\ldots<m_{n} ;\left(s_{1}, \ldots, s_{n}\right) \in \mathbb{C}^{n}\right)$.

In the present paper we introduce a new class of zeta functions $\zeta_{n}^{\mu, s_{1}, \ldots, s_{n}}\left(x_{1}, \ldots, x_{n} ; a_{1}, \ldots, a_{n}\right)$ which is defined by

$$
\begin{aligned}
& \zeta_{n}^{\mu, s_{1}, \ldots, s_{n}}\left(x_{1}, \ldots, x_{n} ; a_{1}, \ldots, a_{n}\right) \\
& =\sum_{m_{1}, \ldots, m_{n}=0}^{\infty} \frac{(\mu)_{m_{1}+\ldots+m_{n}}}{\left(a_{1}+m_{1}\right)^{s_{1}} \ldots\left(a_{n}+m_{n}\right)^{s_{n}}} \frac{x_{1}^{m_{1}}}{m_{1} !} \ldots \frac{x_{n}{ }^{m_{n}}}{m_{n} !}, \\
& \quad\left(\left\{s_{1}, \ldots, s_{n}\right\} \in C ;\left|x_{i}\right|<1, a_{i}>0,(i=1,2, \ldots, n) ;\right. \\
& \left.\mu \in C \backslash \mathbb{Z}_{0}^{-} ; 0 \leq m_{1}<\ldots<m_{n}\right) .
\end{aligned}
$$

Clearly, we have the following relationship

$$
\begin{aligned}
& \zeta_{1}^{1, s}(x ; a)=\Phi(x, s, a), \\
& \text { and } \zeta_{1}^{\mu, s}(x ; a)=\Phi_{\mu}^{*}(x, s, a) .
\end{aligned}
$$

In the case when $s=1,(\forall i=1,2, \ldots, n)$, we have simply 


$$
\begin{aligned}
\zeta_{n}^{\mu, 1, \ldots, 1}\left(x_{1}, \ldots, x_{n} ; a_{1}, \ldots, a_{n}\right) \\
\quad=\sum_{m_{1}, \ldots, m_{n}=0}^{\infty} \frac{(\mu)_{m_{1}+\ldots+m_{n}}}{\left(a_{1}+m_{1}\right) \ldots\left(a_{n}+m_{n}\right)} \frac{x_{1}{ }^{m_{1}}}{m_{1} !} \ldots \frac{x_{n}{ }^{m_{n}}}{m_{n} !}
\end{aligned}
$$

which implies the next result.

Corollary 1.1. Let $\left|x_{1}\right|+\ldots+\left|x_{n}\right|<1, \operatorname{Re}\left(a_{i}\right)>0$, $(\forall i=1,2, \ldots, n)$. Then

$$
\begin{aligned}
& \zeta_{n}^{\mu, 1, \ldots, 1}\left(x_{1}, \ldots, x_{n} ; a_{1}, \ldots, a_{n}\right) \\
& =\left(a_{1}^{-1} \ldots a_{n}^{-1}\right) F_{A}^{(n)}\left[\begin{array}{l}
\mu, a_{1}, \ldots, a_{n} ; \\
a_{1}+1, \ldots, a_{n}+1 \\
x_{1}, \ldots, x_{n}
\end{array}\right]
\end{aligned}
$$

where $F_{A}^{(n)}$ is the Lauricella function of $n$ variables defined by the series (see e.g. [17] and [18]),

$$
\begin{aligned}
& F_{A}^{(n)}\left[a, b_{1}, \ldots, b_{n} ; c_{1}, \ldots, c_{n} ; x_{1}, \ldots, x_{s}\right] \\
& =\sum_{m_{1}, \ldots, m_{n}=0}^{\infty} \frac{(a)_{m_{1}+\ldots+m_{n}}\left(b_{1}\right)_{m_{1}} \ldots\left(b_{n}\right)_{m_{n}}}{\left(c_{1}\right)_{m_{1}} \ldots\left(c_{n}\right)_{m_{1}}} \frac{x_{1}^{m_{1}}}{m_{1} !} \ldots \frac{x_{n}^{m_{n}}}{m_{n} !} .
\end{aligned}
$$

By using the Hankel's contour integral for Gamma function ( for details see e.g. [[19], Section 12.12])

$$
\frac{1}{(a)_{m}}=\frac{\Gamma(a)}{2 i \pi} \int t^{-a-m} e^{-t} d t
$$

we can derive the following interesting formula.

Corollary 1.2. Let $s_{1}=\ldots=s_{n}=1,\left|x_{j}\right|<1,(j=1,2, . ., n)$. Then

$$
\begin{aligned}
& \frac{\Gamma(\mu)}{2 i \pi} \int e^{t} t^{-\mu} \zeta_{n}^{\mu, 1, \ldots, 1}\left(x_{1} t^{-1}, \ldots, x_{n} t^{-1} ; a_{1}, \ldots, a_{n}\right) d t \\
& =\left(a_{1}^{-1} \ldots a_{n}^{-1}\right) \prod_{j=1}^{n}\left\{1 F_{1}\left[\begin{array}{c}
a_{j} ; \\
a_{j}+1 ;
\end{array}\right]\right\} .
\end{aligned}
$$

Proof: The result follow directly from definition (1.9) and the integral representation (1.11).

\section{Integral Representations}

First, by using Eulerian integral formula of the second kind (see e.g. [2]):

$$
\begin{aligned}
& (a+n)^{-s}=\frac{1}{\Gamma(s)} \int_{0}^{\infty} t^{s-1} e^{-(a+n) t} d t, \\
& (\min \{\operatorname{Re}(s), \operatorname{Re}(a+n)\}>0),
\end{aligned}
$$

and the formula(see, e.g., [[20], p.67, Eq (22)]) :

$$
(1-x)^{-\mu}=\sum_{m=0}^{\infty} \frac{(\mu)_{m}}{m !} x^{m}
$$

we should proof the following result.

Theorem 2.1. Let $\operatorname{Re}(\mu)>0, \operatorname{Re}\left(a_{i}\right)>0$ and either

$$
\begin{aligned}
& \left|x_{i}\right|<1, \operatorname{Re}\left(s_{i}\right)>0 \text { or } x_{i}=1, \\
& \operatorname{Re}\left(s_{i}\right)>1\left(\forall i=1,2, \ldots, n, n \in \mathbb{Z}^{+}\right) .
\end{aligned}
$$

Then

$$
\begin{aligned}
& \zeta_{n}^{\mu, s_{1}, \ldots, s_{n}}\left(x_{1}, \ldots, x_{n} ; a_{1}, \ldots, a_{n}\right)=\frac{1}{\Gamma\left(s_{1}\right)} \ldots \frac{1}{\Gamma\left(s_{n}\right)} \\
& \times \int_{0}^{\infty} \ldots \int_{0}^{\infty} t_{1}^{s_{1}-1} \ldots t_{n}^{s_{n}-1} e^{-\left(a_{1} t_{1}+\ldots+a_{n} t_{n}\right)} \\
& \times\left(1-x_{1} e^{-t_{1}}-\ldots-x_{n} e^{-t_{n}}\right)^{-\mu} d t_{1} \ldots d t_{n} .
\end{aligned}
$$

Proof. Denote, for convenience, the right-hand side of equation (2.3) by $I$. Then in view of (2.2), it is easily seen that

$$
\begin{aligned}
I= & \sum_{m_{1}, \ldots, m_{n}=0}^{\infty}(\mu)_{m_{1}+\ldots+m_{n}} \frac{x_{1}^{m_{1}}}{m_{1} !} \ldots \frac{x_{n}^{m_{n}}}{m_{n} !} \frac{1}{\Gamma\left(s_{1}\right)} \ldots \frac{1}{\Gamma\left(s_{n}\right)} \\
& \times \int_{0}^{\infty} \ldots \int_{0}^{\infty} t_{1}^{s_{1}-1} e^{-\left(a_{1}+m_{1}\right) t_{1}} \ldots t_{n}^{s_{n}-1} e^{-\left(a_{n}+m_{n}\right) t_{n}} d t_{1} \ldots d t_{n} .
\end{aligned}
$$

Upon using (2.1) and in view of the definition (1.9), we led finally to the left-hand side of the formula (2.3). Next, by using the contour integral formula [[2], p.14 (4)]:

$$
2 i \sin (\pi s) \Gamma(s)=-\int_{\infty}^{(0+)}(-t)^{s-1} e^{-t} d t, \quad|\arg (-\mathrm{t})| \leq \pi,
$$

one can derive the following contour integral representation.

Theorem 2.2. Let $\operatorname{Re}(\mu)>0, \operatorname{Re}\left(a_{i}\right)>0$ and

$$
\left|\arg \left(-t_{i}\right)\right| \leq \pi,(i=1,2, \ldots, n) .
$$

Then

$$
\begin{aligned}
& \zeta_{n}^{\mu, s_{1}, \ldots, s_{n}}\left(x_{1}, \ldots, x_{n} ; a_{1}, \ldots, a_{n}\right) \\
& =\frac{-\Gamma\left(1-s_{1}\right)}{2 \pi i} \ldots \frac{-\Gamma\left(1-s_{n}\right)}{2 \pi i} \\
& \times \int_{\infty}^{(0+)} \ldots \int_{\infty}^{(0+)}\left(-t_{1}\right)^{s_{1}-1} \ldots\left(-t_{n}\right)^{s_{n}-1} e^{-\left(a_{1} t_{1}+\ldots+a_{n} t_{n}\right)} \\
& \times\left(1-x_{1} e^{-t_{1}}-\ldots-x_{n} e^{-t_{n}}\right)^{-\mu} d t_{1} \ldots d t_{n} .
\end{aligned}
$$

Proof. Denote, for convenience, the right-hand side of equation (2.5) by $I$. Then in view of (2.2), it is easily seen that

$$
\begin{aligned}
& I=\sum_{m_{1}, \ldots, m_{n}=0}^{\infty}(\mu)_{m_{1}+\ldots+m_{n}} \frac{x_{1}^{m_{1}}}{m_{1} !} \\
& \ldots \frac{x_{n} m_{n}}{m_{n} !} \frac{-\Gamma\left(1-s_{1}\right)}{2 \pi i} \ldots \frac{-\Gamma\left(1-s_{n}\right)}{2 \pi i} \\
& \times \int_{\infty}^{(0+)} \ldots \int_{\infty}^{(0+)}\left(-t_{1}\right)^{s_{1}-1} e^{-\left(a_{1}+m_{1}\right) t_{1}} \\
& \ldots\left(-t_{n}\right)^{s_{n}-1} e^{-\left(a_{n}+m_{n}\right) t_{n}} d t_{1} \ldots d t_{n} .
\end{aligned}
$$


Upon using (2.4) and in view of the definition (1.9) with the reflection formula

$$
\Gamma(1-s) \Gamma(s)=\frac{\pi}{\sin (\pi s)}
$$

for Gamma function, we led finally to the left-hand side of the formula (2.5).

Further, we evaluate some definite integrals involving the function $\zeta_{n}^{\mu, s_{1}, \ldots, s_{n}}\left(x_{1}, \ldots, x_{n} ; a_{1}, \ldots, a_{n}\right)$. First, we recall the Eulerian integral formula of first kind (cf. e.g [12]):

$$
\begin{gathered}
B(x, y)=\int_{0}^{1} t^{x-1}(1-t)^{y-1} d t=\frac{\Gamma(x) \Gamma(y)}{\Gamma(x+y)}, \\
\operatorname{Re}(x)>0, \operatorname{Re}(y)>0 .
\end{gathered}
$$

From the term-by-term integration, we can derive the following formula.

Theorem 2.3. Let $\operatorname{Re}(\gamma-\mu)>0$ and $\operatorname{Re}(\mu)>0$. Then

$$
\begin{aligned}
& \zeta_{n}^{\mu, s_{1}, \ldots, s_{n}}\left(x_{1}, \ldots, x_{n} ; a_{1}, \ldots, a_{n}\right) \\
& =\frac{\Gamma(\gamma)}{\Gamma(\gamma-\mu) \Gamma(\mu)} \int_{0}^{1} t^{\mu-1}(1-t)^{\gamma-\mu-1} \\
& \zeta_{n}^{\gamma, s_{1}, \ldots, s_{n}}\left(x_{1} t, \ldots, x_{n} t ; a_{1}, \ldots, a_{n}\right) d t .
\end{aligned}
$$

Proof. Denote for convenience the left-hand side of equation (2.7) by $I$.

Then in view of definition (1.9), it is easily seen that

$$
\begin{aligned}
& I=\sum_{m_{1}, \ldots, m_{n}=0}^{\infty} \frac{(\gamma)_{m_{1}+\ldots+m_{n}}}{\left(a_{1}+m_{1}\right)^{s_{1}} \ldots\left(a_{n}+m_{n}\right)^{s_{n}}} \frac{x_{1}^{m_{1}}}{m_{1} !} \ldots \frac{x_{s}^{m_{n}}}{m_{n} !} \\
& \times \frac{\Gamma(\gamma)}{\Gamma(\gamma-\mu) \Gamma(\mu)} \int_{0}^{1} t^{\mu+m_{1}+\ldots+m_{n}-1}(1-t)^{\gamma-\mu-1} d t .
\end{aligned}
$$

Upon using (2.6) and the relation

$$
\Gamma\left(\mu+m_{1}+. .+m_{n}\right)=(\mu)_{m_{1}+\ldots+m_{n}} \Gamma(\mu),
$$

we are finally led to the right-hand of the relation (2.7).

Finally, we prove the following result.

Theorem 2.4. Let $\operatorname{Re}(\gamma)>\operatorname{Re}(\mu)>0$. Then

$$
\begin{aligned}
& \zeta^{\mu, s_{1}, \ldots, s_{n}}\left(x_{1}, \ldots, x_{n} ; a_{1}, \ldots, a_{n}\right) \\
& =\frac{\Gamma(\gamma)}{\Gamma(\gamma-\mu) \Gamma(\mu)} \int_{0}^{\infty} \frac{w^{\mu-1}}{(1+w)^{\gamma}} \\
& \zeta_{n}^{\gamma, s_{1}, \ldots, s_{n}}\left(\frac{x_{1} w}{1+w}, \ldots, \frac{x_{n} w}{1+w} ; a_{1}, \ldots, a_{n}\right) d w .
\end{aligned}
$$

Proof. Setting $a=\mu+m_{1}+\ldots+m_{n}$ and

$$
b=\gamma+m_{1}+\ldots+m_{n} ; n \in \mathbb{Z}^{+}
$$

in the Eulerian Beta function formula (see, e.g., [[20], p.8, Eq. (45)]):

$$
\begin{aligned}
& B(a, b-a)=\frac{\Gamma(a) \Gamma(b-a)}{\Gamma(b)}=\int_{0}^{\infty} \frac{t^{a-1}}{(1+t)^{b}} d t, \\
& (\operatorname{Re}(b)>\operatorname{Re}(a)>0),
\end{aligned}
$$

we find that

$$
\begin{aligned}
& \frac{(\mu)_{m_{1}+\ldots+m_{n}}}{(\gamma)_{m_{1}+\ldots+m_{n}}}=\frac{\Gamma(\gamma)}{\Gamma(\gamma-\mu) \Gamma(\mu)} \int_{0}^{\infty} \frac{w^{\mu+m_{1}+\ldots+m_{n}-1}}{(1+w)^{\gamma+m_{1}+\ldots+m_{n}}} d w, \\
& \left(\operatorname{Re}(\gamma)>\operatorname{Re}(\mu)>0 ; m_{i} \in \mathbb{N}_{0},(i=1,2, \ldots, n)\right),
\end{aligned}
$$

which, by appealing to the definition (1.9), immediately yields the assertion (2.8).

\section{Operational Relations}

The multiple Zeta function $\zeta_{n}^{\mu, s_{1}, \ldots, s_{n}}$ satisfies some operational relations. Fortunately these properties of $\zeta_{n}^{\mu, s_{1}, \ldots, s_{n}}$ be developed directly from the definition (1.9). First, by recalling the familiar derivative formula from calculus in terms of the gamma function [8]

$$
D_{x}^{m} x^{n}=\frac{\Gamma(n+1)}{\Gamma(n-m+1)} x^{n-m}, n-m \geq 0, D_{x}=\frac{d}{d x},
$$

where $m \in \mathbb{N}$, we aim now to derive the following differential relations for $\zeta_{n}^{\mu, s_{1}, \ldots, s_{n}}$.

Theorem 3.1. Let $k_{i} \in \mathbb{N},(i=1,2, \ldots, n)$. Then

$$
\begin{aligned}
& \prod_{i=}^{n}\left\{\begin{array}{c}
k_{i} \\
x_{i}
\end{array}\right\} \zeta_{n}^{\mu, s_{1}, \ldots, s_{n}}\left(x_{1}, \ldots, x_{n} ; a_{1}, \ldots, a_{n}\right) \\
& =(\mu)_{k_{1}+\ldots+k_{n}} \zeta_{n}^{\mu+k_{1}+\ldots+k_{n}, s_{1}, \ldots, s_{n}}\left(\begin{array}{l}
x_{1}, \ldots, x_{n} ; \\
a_{1}+k_{1}, \ldots, a_{n}+k_{n}
\end{array}\right) .
\end{aligned}
$$

Proof. By starting from the left-hand side of (3.2) and in view of (1.9) and by using the relation (3.1), we get:

$$
\begin{aligned}
& \prod_{i=}^{n}\left\{D_{x_{i}}^{k_{i}}\right\} \zeta_{n}^{\mu, s_{1}, \ldots, s_{n}}\left(x_{1}, \ldots, x_{n} ; a_{1}, \ldots, a_{n}\right) \\
& =\sum_{m_{1}, \ldots, m_{n}=0}^{\infty}\left(\begin{array}{l}
\frac{(\mu)_{m_{1}+\ldots+m_{n}}}{\left(a_{1}+m_{1}\right)^{s_{1}} \ldots\left(a_{n}+m_{n}\right)^{s_{n}}} \\
\frac{x_{1}^{m_{1}-k_{1}}}{\left(m_{1}-k_{1}\right) !} \ldots \frac{x_{n}^{m_{n}-k_{n}}}{\left(m_{n}-k_{n}\right) !}
\end{array}\right) .
\end{aligned}
$$

Now, letting $m_{i} \rightarrow m_{i}+k_{i},(i=1,2, \ldots, n)$ in (3.3) using the formula $(\mu)_{m+k}=(\mu)_{k}(\mu+k)_{m}$ and considering the definition (1.9), we get the right-hand side of formula (3.2).

Theorem 3.2. Let

$$
k_{i} \in \mathbb{N}, s_{i} \neq 0,-1,-2, \ldots \ldots,(i=1,2, \ldots, n) .
$$

Then

$$
\begin{aligned}
& \prod_{i=1}^{n}\left\{\frac{\partial^{k_{i}}}{\partial a_{i}^{k_{i}}}\right\} \zeta_{n}^{\mu, s_{1}, \ldots, s_{n}}\left(x_{1}, \ldots, x_{n} ; a_{1}, \ldots, a_{n}\right) \\
& =\prod_{i=1}^{n}\left\{(-1)^{k_{i}}\left(s_{i}\right)_{k_{i}}\right\} \\
& \times \zeta_{n}^{\mu, s_{1}+k_{1}, \ldots, s_{n}+k_{n}}\left(x_{1}, \ldots, x_{n} ; a_{1}, \ldots, a_{n}\right) .
\end{aligned}
$$


Proof. By starting from the left-hand side of (3.4) and in view of (1.9) and by using the relation

$$
\frac{\partial^{k}}{\partial a^{k}}(a+m)^{-s}=(-1)^{k}(s)_{k}(a+m)^{-s-k},
$$

we get:

$$
\prod_{i=1}^{n}\left\{D_{a_{i}}^{k_{i}}\right\} \zeta_{n}^{\mu, s_{1}, \ldots, s_{n}}\left(x_{1}, \ldots, x_{n} ; a_{1}, \ldots, a_{n}\right)
$$

$=\prod_{i=1}^{n}\left\{(-1)^{k_{i}}\left(s_{i}\right)_{k_{i}}\right\}$

$\sum_{m_{1}, \ldots, m_{n}=0}^{\infty} \frac{(\mu)_{m_{1}+\ldots+m_{n}}}{\left(a_{1}+m_{1}\right)^{s_{1}+k_{1}} \ldots\left(a_{n}+m_{n}\right)^{s_{n}+k_{n}}} \frac{x_{1}^{m_{1}}}{m_{1} !} \ldots \frac{x_{n}^{m_{n}}}{m_{n} !}$.

Now, considering the definition (1.9), we get the right-hand side of formula (3.4).

Closely associated with the derivative of the gamma function is the digamma function defined by $[[21]$, p.74(2.51)]:

$$
\psi(x)=\frac{d}{d x} \ln \Gamma(x)=\frac{\Gamma^{\prime}(x)}{\Gamma(x)},(x) \neq 0,-1,-2, \ldots .
$$

Now, we wish to establish the derivative of the function $\zeta_{n}^{\mu, s_{1}, \ldots, s_{n}}$ with respect to the parameters $\mu$.

Theorem 3.3. Let $\operatorname{Re}(\mu) \in \mathbb{C} \backslash \mathbb{Z}_{0}^{-}$. Then

$$
\begin{aligned}
& \frac{\partial}{\partial \mu} \zeta_{n}^{\mu, s_{1}, \ldots, s_{n}}\left(x_{1}, \ldots, x_{n} ; a_{1}, \ldots, a_{n}\right) \\
& =\sum_{m_{1}, \ldots, m_{n}=0}^{\infty} \frac{(\mu)_{m_{1}+\ldots+m_{n}}}{\left(a_{1}+m_{1}\right)^{s_{1}} \ldots\left(a_{n}+m_{n}\right)^{s_{n}}} \frac{x_{1}^{m_{1}}}{m_{1} !} \ldots \frac{x_{s}^{m_{n}}}{m_{n} !} \\
& \times\left[\psi\left(\mu+m_{1}+\ldots+m_{n}\right)-\psi(\mu)\right] .
\end{aligned}
$$

Proof. By starting from the left-hand side of formula (3.6) and by using the relation (3.5), then according to the result by using:

$$
\frac{d}{d \mu}(\mu)_{n}=\frac{d}{d \mu}\left[\frac{\Gamma(\mu+n)}{\Gamma(\mu)}\right]=(\mu)_{n}[\psi(\mu+n)-\psi(\mu)],
$$

we obtain the right-hand side of formula (3.6).

Next, let us recall the definition of the Weyl fractional derivative of exponential function $e^{-a t}, a>0$ of order $v$ in the form (see [[22], p.248(7.4)] ):

$$
D_{t}^{v} e^{-a t}=a^{v} e^{-a t} \text {, }
$$

( $v$ not restricted to be positive integer).:

We now proceed to find the fractional derivative of the function $\zeta_{n}^{\mu, s_{1}, \ldots, s_{n}}$ with respect to $s$.

Theorem 3.4. Let $v_{i}>0,(i=1,2, \ldots, n)$. Then

$$
\begin{aligned}
& \prod_{i=1}^{n}\left\{D_{s_{i}}^{v_{i}}\right\} \zeta_{n}^{\mu, s_{1}, \ldots, s_{n}}\left(x_{1}, \ldots, x_{n} ; a_{1}, \ldots, a_{n}\right) \\
& =\sum_{m_{1}, \ldots, m_{n}=0}^{\infty} \frac{(\mu)_{m_{1}+\ldots+m_{n}}}{\left(a_{1}+m_{1}\right)^{s_{1}} \ldots\left(a_{n}+m_{n}\right)^{s_{n}}} \frac{x_{1}^{m_{1}}}{m_{1} !} \ldots \frac{x_{n}^{m_{n}}}{m_{n} !} \\
& \times \prod_{i=1}^{n}\left\{\left[\log \left(a_{i}+m_{i}\right)\right]^{v_{i}}\right\} .
\end{aligned}
$$

Proof. Since $(a+m)^{(-s)}=e^{(-s \log (a+m))}$, we have

$$
\begin{aligned}
& \zeta_{n}^{\mu, s_{1}, \ldots, s_{n}}\left(x_{1}, \ldots, x_{n} ; a_{1}, \ldots, a_{n}\right)=\sum_{m_{1}, \ldots, m_{n}=0}^{\infty}(\mu)_{m_{1}+\ldots+m_{n}} \\
& \times \frac{x_{1}^{m_{1}}}{m_{1} !} \ldots \frac{x_{n}^{m_{n}}}{m_{n} !} e^{-s_{1} \log \left(a_{1}+m_{1}\right)} \ldots e^{-s_{n} \log \left(a_{n}+m_{n}\right)}
\end{aligned}
$$

The desired result now follows by applying the formula (3.8) to the above identity.

\section{Series Expansions}

First we derive the following basic sums of series

Theorem 4.1. Let $s_{i} \neq 0,1,2, \ldots \ldots,(\forall i=1,2, \ldots, n)$. Then

$$
\begin{aligned}
& \sum_{k_{1}, \ldots, k_{n}=0}^{\infty} \zeta_{n}^{\mu, s_{1}-k_{1}, \ldots, s_{n}-k_{n}}\left(\begin{array}{c}
x_{1}, \ldots, x_{n} ; \\
a_{1}, \ldots, a_{n}
\end{array}\right) \frac{y_{1}{ }^{k}}{k_{1} !} \ldots \frac{y_{n}{ }^{k_{n}}}{k_{n} !} \\
& =\exp \left(\sum_{i=1}^{n} a_{i} y_{i}\right) \zeta_{n}^{\mu, s_{1}, \ldots, s_{n}}\left(x_{1} e^{y_{1}}, \ldots, x_{n} e^{y_{n}} ; a_{1}, \ldots, a_{n}\right) \text {. }
\end{aligned}
$$

Proof. If in formula (1.9), we replace $s_{i}$ by $s_{i}-k_{i}$, $(i=1,2, \ldots, n)$, multiply throughout by $\frac{y_{1}^{k_{1}}}{k_{1} !} \ldots \frac{y_{n}^{k_{n}}}{k_{n} !}$ and then sums up, we get (4.1).

Further, from definition (1.9) we easily have the following interesting series relation.

Theorem 4.2. Let $\left|x_{i}\right|<1,\left\{\left|y_{i}\right|<\left|a_{i}\right|\right\}, \quad s_{i} \neq 0,-1,-2, \ldots$, $(\forall i=1,2, \ldots, n)$. Then

$$
\begin{aligned}
& \sum_{k_{1}, \ldots, k_{n}=0}^{\infty} \zeta_{n}^{\mu, s_{1}+k_{1}, \ldots, s_{n}+k_{n}}\left(x_{1}, \ldots, x_{n} ; a_{1}, \ldots, a_{n}\right) \prod_{i=1}^{n}\left\{\left(s_{i}\right)_{k_{i}} \frac{y_{i}^{k_{i}}}{k_{i} !}\right\} \\
= & \zeta_{n}^{\mu, s_{1}, \ldots, s_{n}}\left(x_{1}, \ldots, x_{n} ; a_{1}-y_{1}, \ldots, a_{n}-y_{n}\right), \\
= & \exp \left(-\sum_{i=1}^{n} \boldsymbol{y}_{\boldsymbol{i}} \frac{\partial}{\partial \boldsymbol{a}_{\boldsymbol{i}}}\right) \zeta_{n}^{\mu, s_{1}, \ldots, s_{n}}\left(x_{1}, \ldots, x_{n} ; a_{1}, \ldots, a_{n}\right) .
\end{aligned}
$$

Proof. Since

$$
\begin{aligned}
(a+m-y)^{-s}= & (a+m)^{-s}\left(1-\frac{y}{a+m}\right)^{-s} \\
& =\sum_{k=0}^{\infty} \frac{(s)_{k}}{(a+m)^{s+k}} \frac{y^{k}}{k !}
\end{aligned}
$$

it is easily seen that :

$$
\begin{aligned}
& \zeta_{n}^{\mu, s_{1}, \ldots, s_{n}}\left(x_{1}, \ldots, x_{n} ; a_{1}-y_{1}, \ldots, a_{n}-y_{n}\right) \\
& =\sum_{k_{1}, \ldots, k_{n}=0}^{\infty} \prod_{i=1}^{n}\left\{\left(s_{i}\right)_{k_{i}} \frac{y_{i}^{k_{i}}}{k_{i} !}\right\} \\
& \sum_{m_{1}, \ldots, m_{n}=0}^{\infty} \frac{(\mu)_{m_{1}+\ldots+m_{n}}}{\left(a_{1}+m_{1}\right)^{s_{1}+k_{1}} \ldots\left(a_{n}+m_{n}\right)^{s_{n}+k_{n}}} \frac{x_{1}^{m_{1}}}{m_{1} !} \ldots \frac{x_{n}^{m_{n}}}{m_{n} !} .
\end{aligned}
$$


The desired result (4.2) now follows by using definition (1.9). Also, by right-hand formula (4.3) and using (3.4) we get to left-hand side of formula (4.3).

Theorem 4.3. Let $s_{i} \neq 0,-1,-2, \ldots \ldots, \quad(\forall i=1,2, \ldots, n)$. Then

$$
\begin{aligned}
& \sum_{k_{1}, \ldots, k_{n}=0}^{\infty}(\alpha)_{k_{1}+\ldots+k_{n}} \zeta_{n}^{\mu, s_{1}+k_{1}, \ldots, s_{n}+k_{n}}\left(x_{1}, \ldots, x_{n} ; a_{1}, \ldots, a_{n}\right) \\
\times & \prod_{i=1}^{n}\left\{\left(\beta_{i}\right)_{k_{i}} \frac{y_{i}^{k_{i}}}{k_{i} !}\right\} \\
= & F_{A}^{(n)}\left[\alpha, \beta_{1}, \ldots, \beta_{n} ; s_{1}, \ldots, s_{n} ;-y_{1} \frac{\partial}{\partial a_{1}}, \ldots,-y_{n} \frac{\partial}{\partial a_{n}}\right] \\
\times & \zeta_{n}^{\mu, s_{1}, \ldots, s_{n}}\left(x_{1}, \ldots, x_{n} ; a_{1}, \ldots, a_{n}\right),
\end{aligned}
$$$$
\text { where }\left|-y_{1} \frac{\partial}{\partial a_{1}}\right|+\ldots+\left|-y_{n} \frac{\partial}{\partial a_{n}}\right|<1 \text {; }
$$$$
\sum_{k_{1}, \ldots, k_{n}=0}^{\infty} \frac{1}{(\gamma)_{k_{1}+\ldots+k_{n}}} \zeta_{n}^{\mu, s_{1}+k_{1}, \ldots, s_{n}+k_{n}}\left(x_{1}, \ldots, x_{n} ; a_{1}, \ldots, a_{n}\right)
$$$$
\times \prod_{i=1}^{n}\left\{\left(\alpha_{i}\right)_{k_{i}} \frac{y_{i}^{k_{i}}}{k_{i} !}\right\}
$$$$
=F_{B}^{(n)}\left[\alpha_{1}, \ldots, \alpha_{n}, s_{1}, \ldots, s_{n} ; \gamma ;-y_{1} \frac{\partial}{\partial a_{1}}, \ldots,-y_{n} \frac{\partial}{\partial a_{n}}\right]
$$$$
\times \zeta_{n}^{\mu, s_{1}, \ldots, s_{n}}\left(x_{1}, \ldots, x_{n} ; a_{1}, \ldots, a_{n}\right) \text {, }
$$$$
\text { where } \max \left\{\left|-y_{1} \frac{\partial}{\partial a_{1}}\right|+\ldots+\left|-y_{n} \frac{\partial}{\partial a_{n}}\right|\right\}<1 \text {; }
$$$$
\sum_{k_{1}, \ldots, k_{n}=0}^{\infty}(\alpha)_{k_{1}+\ldots+k_{n}}(\beta)_{k_{1}+\ldots+k_{n}}
$$$$
\times \zeta_{n}^{\mu, s_{1}+k_{1}, \ldots, s_{n}+k_{n}}\left(x_{1}, \ldots, x_{n} ; a_{1}, \ldots, a_{n}\right) \prod_{i=1}^{n}\left\{\frac{y_{i}^{k_{i}}}{k_{i} !}\right\}
$$$$
=F_{C}^{(n)}\left[\alpha, \beta ; s_{1}, \ldots, s_{n} ;-y_{1} \frac{\partial}{\partial a_{1}}, \ldots,-y_{n} \frac{\partial}{\partial a_{n}}\right]
$$$$
\times \zeta_{n}^{\mu, s_{1}, \ldots, s_{n}}\left(x_{1}, \ldots, x_{n} ; a_{1}, \ldots, a_{n}\right), ?
$$$$
\text { where } \sqrt{\left|-y_{1} \frac{\partial}{\partial a_{1}}\right|}+\ldots+\sqrt{\left|-y_{n} \frac{\partial}{\partial a_{n}}\right|}<1 \text {; and }
$$

$$
\begin{aligned}
& \sum_{k_{1}, \ldots, k_{n}=0}^{\infty} \frac{(\alpha)_{k_{1}+\ldots+k_{n}}}{(\gamma)_{k_{1}+\ldots+k_{n}}} \zeta_{n}^{\mu, s_{1}+k_{1}, \ldots, s_{n}+k_{n}}\left(x_{1}, \ldots, x_{n} ; a_{1}, \ldots, a_{n}\right) \\
\times & \prod_{i=1}^{n}\left\{\frac{y_{i}^{k_{i}}}{k_{i} !}\right\} \\
= & F_{D}^{(n)}\left[\alpha, s_{1}, \ldots, s_{n} ; \gamma ;-y_{1} \frac{\partial}{\partial a_{1}}, \ldots,-y_{n} \frac{\partial}{\partial a_{n}}\right] \\
\times & \zeta_{n}^{\mu, s_{1}, \ldots, s_{n}}\left(x_{1}, \ldots, x_{n} ; a_{1}, \ldots, a_{n}\right) .
\end{aligned}
$$

where $F_{A}^{(n)}, F_{B}^{(n)}, F_{C}^{(n)}$ and $F_{D}^{(s)}$ are the Lauricella's hypergeometric functions of $n$-variables (see [[18], p.60, Eq (1), (2), (3), (4)]).

Proof. We refer to the proof of Theorem 4.2.
Theorem 4.4. Let $\left|y_{i}\right|<1,(i=1,2, \ldots, n)$. Then

$$
\begin{aligned}
& \sum_{k_{1}, \ldots, k_{n}=0}^{\infty}(\mu)_{k_{1}+\ldots+k_{n}} \\
& \zeta_{n}^{\mu+k_{1}+\ldots+k_{n}, s_{1}, \ldots, s_{n}}\left(x_{1}, \ldots, x_{n} ; a_{1}+k_{1}, \ldots, a_{n}+k_{n}\right) \prod_{i=1}^{n}\left\{\frac{y_{i}^{k_{i}}}{k_{i} !}\right\} \\
&=\zeta_{n}^{\mu, s_{1}, \ldots, s_{n}}\left(x_{1}+y_{1}, \ldots, x_{n}+y_{n} ; a_{1}, \ldots, a_{n}\right) . ?
\end{aligned}
$$

Proof. By starting from the right-hand side of (4.8) and in view of (1.9) and by using the relation

$$
\sum_{m=0}^{\infty} \frac{(\mu)_{m}}{(a+m)^{s}} \frac{(x+y)^{m}}{m !}=\sum_{k=0}^{\infty} \sum_{m=0}^{\infty} \frac{(\mu)_{m+k}}{(a+m+k)^{s}} \frac{x^{m}}{m !} \frac{y^{k}}{k !},
$$

we get the left-hand side of formula (4.8).

Theorem 4.5. Let $\left|x_{i}\right|<1,\left|y_{i}\right|<\left|a_{i}\right|$ and $\left|t_{i}\right|<\left|a_{i}\right|$; $(i=1,2, \ldots, n)$. Then

$$
\begin{aligned}
& \sum_{k_{1}, \ldots, k_{n}=0}^{\infty} \zeta_{n}^{\mu, s_{1}+k_{1}, \ldots, s_{n}+k_{n}}\left(x_{1}, \ldots, x_{n} ; a_{1}-t_{1}, \ldots, a_{n}-t_{n}\right) \\
\times & \prod_{i=1}^{n}\left\{\left(b_{i}\right)_{k_{i}} \frac{y_{i}^{k_{i}}}{k_{i} !}\right\}=\sum_{m_{1}, \ldots, m_{n}=0}^{\infty}(\mu)_{m_{1}+\ldots+m_{n}} \\
\times & \prod_{i=1}^{n}\left\{\frac{x_{i}^{m_{i}}}{\left(a_{i}+m_{i}\right)^{s_{i}} m_{i} !} F_{2}\left[s_{i}, b_{i}, 1 ; s_{i}, 1 ; \frac{y_{i}}{a_{i}+m_{i}}, \frac{t_{i}}{a_{i}+m_{i}}\right]\right\},
\end{aligned}
$$

where $F_{2}$ is the Appell's function of two variables defined by the series [18]

$$
F_{2}\left[a, b, b^{\prime} ; \mathrm{c}, c^{\prime} ; x, y\right]=\sum_{m, n=0}^{\infty} \frac{(a)_{m+n}(b)_{m}\left(b^{\prime}\right)_{n}}{(c)_{m}\left(c^{\prime}\right)_{n}} \frac{x^{m}}{m !} \frac{y^{n}}{n !} .
$$

Proof. By starting from the right-hand side of (4.9) and in view of (1.9), we get

$$
\begin{aligned}
& \sum_{k_{1}, \ldots, k_{n}=0}^{\infty} \zeta_{n}^{\mu, s_{1}+k_{1}, \ldots, s_{n}+k_{n}}\left(x_{1}, \ldots, x_{n} ; a_{1}-t_{1}, \ldots, a_{n}-t_{n}\right) \\
& \prod_{i=1}^{n}\left\{\left(b_{i}\right)_{k_{i}} \frac{y_{i}^{k_{i}}}{k_{i} !}\right\}=\sum_{k_{1}, \ldots, k_{n}=0 m_{1}, \ldots, m_{n}=0}^{\infty} \sum_{m_{1}+\ldots+m_{n}}^{\infty}(\mu)_{i=1}\left\{\frac{\left(b_{i}\right)_{k_{i}}}{\left(a_{i}+m_{i}-t_{i}\right)^{s_{i}+k_{i}}} \frac{x_{i}^{m_{i}}}{m_{i} !} \frac{y_{i}^{k_{i}}}{k_{i} !}\right\} .
\end{aligned}
$$

Since

$$
\begin{aligned}
& \sum_{k=0}^{\infty}(b)_{k} \frac{y^{k}}{k !}(a+m-t)^{-(s+k)} \\
& =\sum_{k=0}^{\infty}(b)_{k} \frac{y^{k}}{k !}(a+m)^{-(s+k)}\left(1-\frac{t}{a+m}\right)^{-(s+k)} \\
& =\sum_{k=0}^{\infty}(b)_{k} \frac{(y / a+m)^{k}}{k !} \sum_{r=0}^{\infty} \frac{(s+k)_{r}}{(a+m)^{s}} \frac{(t / a+m)^{r}}{r !}, ? \\
& =(a+m)^{s} \sum_{k, r=0}^{\infty} \frac{(s)_{k+r}(b)_{k}(1)_{r}}{(1)_{r}(s)_{k}} \frac{(t / a+m)^{r}}{r !} \frac{(y / a+m)^{k}}{k !} .
\end{aligned}
$$


Hence, the right-hand side of formula (4.9) follows.

\section{References}

[1] Chaudhry M. A. and Zubair S. M., On a Class of Incomplete Gamma Functions with Applications, Chapman and Hall, (CRC Press Company), Boca Raton, London, New York and Washington, D. C., 2001.

[2] Erdélyi,A., Magnus,W., Oberhettinger, F. and Tricomi, F.G. Higher Transcendental Functions, Vol.I, McGraw - Hill book inc. New York, Toronto and London, 1953.

[3] Jankov, D., Pogány T. K. and Saxena, R. K. An extended general Hurwitz-Lerch Zeta function as a Mathieu $(a, \lambda)$-series, Appl. Math. Lett., 24, 1473-1476,2011.

[4] Bin-Saad Maged G. , "Sums and partial sums of double power series associated with the generalized zeta function and their $\mathrm{N}$ fractional calculus", Math. J. Okayama University, 49, 37-52, 2007.

[5] Bin-Saad Maged G.,"Hypergeometric Series Associated with the Hurwitz-Lerch Zeta Function", Acta Math. Univ. Comenianae, 2, 269-286, 2009.

[6] Bin-Saad Maged G. and Al Gonah, A.A., On hypergeometric type generating functions associated with generalized zeta function, Acta Math. Univ. Comenianae, 2, 253-266, 2006

[7] Bin-Saad Maged G., Pathan M. A. and Hanballa Amani M.,On power series associated with generalized multiple zeta function, Math. Sci. Res. J. 17(10) 279-291, 2013.

[8] Choi J., Multiple gamma function and their applications, in Proc Internat. Conf. on Analysis (editied by Y.C. Kim), Yeungnam Univeristy, Korea, 73-84,1996.

[9] Choi J., Jang D. S. and Srivastava H. M., A generalization of the Hurwitz-Lerch Zeta function, Integral Transforms Spec. Funct. 19 65-79, 2008.

[10] Goyal, S. and Laddha, R.K. , On the Generalized Riemann Zeta Funcion and the Generalized Lambert Transform, Ganita Sandesh, 11, 99-108, 1997.
[11] Lin S. D. and Srivastava H. M., Some families of the HurwitzLerch Zeta functions and associated fractional derivative and other integral representations, Appl. Math. Comput. 154, 725-733, 2004.

[12] Srivastava, H. M., M. J. Luo and. Raina, R. K, New results involving a class of generalized Hurwitz-Lerch Zeta functions and their applications, Turkish J. Anal. Number Theory 1(1), 26-35, 2013.

[13] Srivastava, H. M., R. K. Saxena, T. K. Pogány and R. Saxena, Integral and computational representations of the extended Hurwitz-Lerch Zeta function, Integral Transforms Spec. Funct. 22(7), 487-506, 2011.

[14] Srivastava, H. M., D. Jankov, Pogány, D., T. K. and R. K. Saxena, Two-sided inequalities for the extended Hurwitz-Lerch Zeta function, Comput. Math. Appl. 62 (2011), 516-522

[15] Kamano K., The multiple Hurwitz Zeta function and a generalization of Lerch's formula, Tokyo J. Math. 29, 61-73, 2006.

[16] Matsumoto, K., The analytic continuation and the asymptonic behaviour of certain multiple zeta-functions I, J. Number Theory $101,223-243,2003$

[17] Srivastava, H. M. and Karlsson, P. W., Multiple Gaussian Hypergeometric Series, Halsted Press, Brisbane, London, New York, 1985.

[18] Srivastava, H. M. and Manoch, H. L., A treatise on Generating Functions, Halsted Press, Brisbane, London, New York, 1984.

[19] Whittaker, E. T. and Watson, G. N., A course of modern Analysis, Fourth Edition, Cambridge Uni. Press, 1952.

[20] Srivastava H. M. and Choi J., Zeta and q-Zeta Functions and Associate Series and Integrals, Elsevier Science, Publishers, Amsterdam, London and New York, 2012.

[21] Andrews, L.C., Special Functions for Engineers and Applied Mathematician, Mac-Millan, New York, 1985.

[22] Miller, K.S. and Rose, B., An introduction to The Fractional Calculus and Fractional Differential Equations, New York, 1993.

[23] Lauricella, G., Sulle funzioni ipergeometriche a piu variabili. Rend. Circ. Mat. Palermo 7, 111-158, 1893 